\title{
An approach to verification and validation of MHD codes for fusion applications
}

\author{
S. Smolentsev ${ }^{1, *}$, S. Badia ${ }^{2,3}$, R. Bhattacharyay ${ }^{4}$, L. Bühler ${ }^{5}$, L. Chen ${ }^{10}$, Q. Huang ${ }^{6}$, \\ H.-G. Jin ${ }^{7}$, D. Krasnov ${ }^{8}$, D.-W. Lee ${ }^{7}$, E. Mas de les Valls ${ }^{2,3}$, C. Mistrangelo ${ }^{5}$, R. \\ Munipalli' ${ }^{9}$, M.-J. Ni ${ }^{10}$, D. Pashkevich ${ }^{11}$, A. Patel $^{3}$, G. Pulugundla ${ }^{1}$, P. Satyamurthy ${ }^{12}$, \\ A. Snegirev ${ }^{11}$, V. Sviridov ${ }^{13}$, P. Swain ${ }^{12}$, T. Zhou ${ }^{6}$, O. Zikanov ${ }^{14}$ \\ ${ }^{1}$ University of California, Los Angeles, USA \\ ${ }^{2}$ Centre Internacional de Mètodes Numèrics en Enginyeria, Barcelona, Spain \\ ${ }^{3}$ Universitat Politècnica de Catalunya - Barcelona Tech, Spain \\ ${ }^{4}$ Institute for Plasma Research, Gandhinagar, Gujarat, India \\ ${ }^{5}$ Karlsruhe Institute of Technology, Germany \\ ${ }^{6}$ Institute of Nuclear Energy Safety Technology, Chinese Academy of Sciences, Hefei, \\ Anhui, China \\ ${ }^{7}$ Korea Atomic Energy Research Institute, Daejeon, South Korea \\ ${ }^{8}$ Technische Universität Ilmenau, Germany \\ ${ }^{9}$ HyPerComp, Westlake Village, USA \\ ${ }^{10}$ University of Chinese Academy of Sciences, Beijing, China \\ ${ }^{11}$ St.Petersburg State Polytechnical University, Russia \\ ${ }^{12}$ Bhabha Atomic Research Center, India \\ ${ }^{13}$ Moscow Power Engineering Institute, Russia \\ ${ }^{14}$ University of Michigan - Dearborn, USA
}

\begin{abstract}
We propose a new activity on verification and validation (V\&V) of MHD codes presently employed by the fusion community as a predictive capability tool for liquid metal cooling applications, such as liquid metal blankets. The important steps in the development of MHD codes starting from the 1970s are outlined first and then basic MHD codes, which are currently in use by designers of liquid breeder blankets, are reviewed. A benchmark database of five problems has been proposed to cover a wide range of MHD flows from laminar fully developed to turbulent flows, which are of interest for fusion applications: (A) 2D fully developed laminar steady MHD flow, (B) 3D laminar, steady developing MHD flow in a non-uniform magnetic field, (C) quasitwo-dimensional MHD turbulent flow, (D) 3D turbulent MHD flow, and (E) MHD flow with heat transfer (buoyant convection). Finally, we introduce important details of the proposed activities, such as basic V\&V rules and schedule. The main goal of the present paper is to help in establishing an efficient V\&V framework and to initiate benchmarking among interested parties. The comparison results computed by the codes against analytical solutions and trusted experimental and numerical data as well as code-to-code comparisons will be presented and analyzed in companion paper/papers.
\end{abstract}

Keywords: Blanket; Liquid metal magnetohydrodynamics; Computer code

${ }^{*}$ Corresponding author. Tel.: +1 3107945354 .

E-mail address: sergey@fusion.ucla.edu (S. Smolentsev). 


\section{Introduction}

This paper is a follow up of a talk given by the first author at the IEA Liquid Breeder Blanket (LBB) Workshop in Barcelona, Spain on Sep. 21, 2013 about a new initiative aiming at verification and validation $(\mathrm{V} \& \mathrm{~V})$ of $\mathrm{MHD}$ codes, which have recently been used as a design/analysis tool for fusion applications, first of all, for liquid metal (LM) breeding blankets. There were about 40 attendees from the US, EU, Japan, China, Russia, India and Korea involved in liquid breeder activities in their countries, in particular in development of magnetohydrodynamic (MHD) codes. As a result of the subsequent round-table discussion, the participants had established a work group, agreed about basic $\mathrm{V} \& \mathrm{~V}$ rules and the schedule and finally recommended a set of test cases for the upcoming benchmark activities.

The paper outlines major steps in development of MHD codes starting from the 1970s, summarizes the most important goals of the proposed test activities, introduces major MHD codes presently employed by the fusion community, selects five benchmark cases for laminar and turbulent MHD flows, and gives recommendations on how the testing of the codes could be organized among the participants. We also review earlier code benchmarking activities [1] for hydrodynamic flows. The main goal of the present paper is therefore to help in establishing an efficient framework for V\&V of MHD codes for fusion applications. Results of the proposed testing will be reported in the next

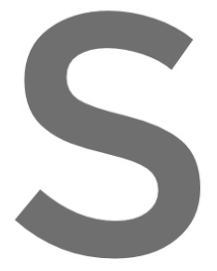
companion paper/papers when the test cases are complited by the performers. The
expected duration of the proposed test studies is about one year from the moment when
this paper is published.
This publication, as the title implies, is primarily concerned with verification and
validation. These two terms are often confused especially when applied to conputationa fluid dynamics (CFD). Based on the AIAA glossary for CFD [2], verification is defined

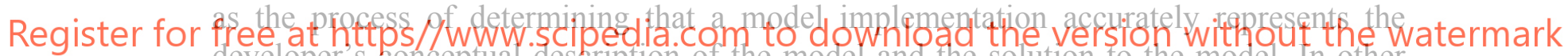
developer's conceptual description of the model and the solution to the model. In other words, verification can be described as "solving the equations right". Validation is defined as the process of determining the degree to which a model is an accurate representation of the real world from the perspective of the intended uses of the model. Validation has also been described as "solving the right equations". CFD benchmarking is the key approach used to verify and validate a code, which includes the comparison of numerical data produced by a code with reference values, which are commonly accepted by the CFD community. Code benchmarking can include comparisons with experimental data or testing against trusted numerical data or analytical solutions.

The main objectives of the proposed activities on V\&V of MHD codes for fusion applications, as agreed among the participants of the LBB workshop in Barcelona, are the following:

- To help the code developers to improve their computational tools via code-tocode comparisons as well as benchmarking against available analytical solutions and existing and near-future experimental data and also against trusted numerical data.

- To establish a benchmark database.

- To share information about recent code development. 
- To attract more attention in the fusion community to problems and difficulties in developing fusion-relevant MHD codes as a tool for LM blanket design and analysis.

- To educate current and potential users about code capabilities and limitations to encourage "critical thinking" and "thoughtful approach" when applying MHD codes to fusion problems.

- To start developing a policy on the control of computational quality, in particular to provide reviewers of fusion journals with a set of criteria by which the reviewers can judge the quality of publications in the area of computational MHD (CMHD).

\section{MHD modeling background}

At present, there is a critical demand in the fusion community for effective MHD codes that could be (i) used for LBB design and analysis and (ii) implemented in integrated modeling tools to address multiple physical effects in blanket flows. Unfortunately, only a few large MHD codes are presently available for blanket applications, which are, in fact, less mature than ordinary CFD codes. Their applicability to the full-scale fusion problems is still limited by the magnetic field strength, flow velocity and geometrical complexity. These limitations can be illustrated with a simple

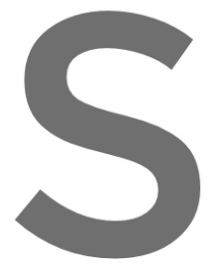
diagram (Fig. 1), the dimensionless $H a=B_{0} L \sqrt{\sigma / v \rho}$ sectional dimension of the flow-carrying working fluid, and $v$ and $\rho$ are the fluid kinematic viscosity and density. For flows in a

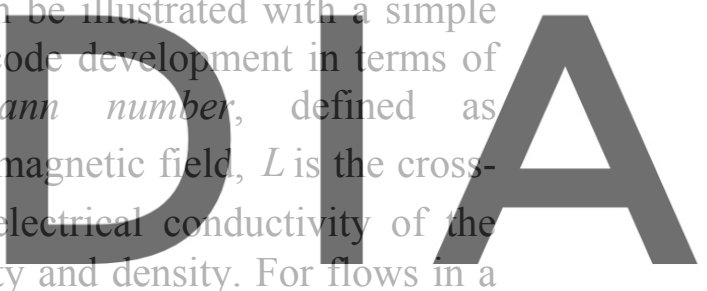

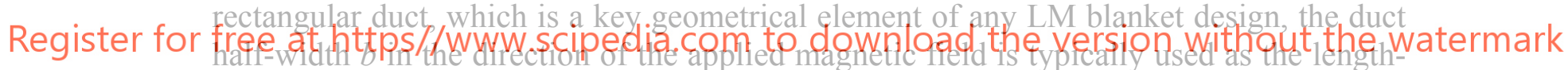
scale: $L=b$. Hartmann number squared is also interpreted as the ratio between MHD and viscous forces. MHD computations were pioneered in the 1970 s but at that time were limited to Hartmann numbers of a few tens [3]. The computations progressed quickly over the next three decades reaching Hartmann numbers on the order of hundreds in the late 1980s (e.g., [4]) and a few thousands recently [5]. Significant acceleration in MHD computations can be seen at around 2005 due to development of a new consistent and conservative scheme [6]. However, the progress has been different between simple geometry flows (e.g. in a straight rectangular duct) and more complex flows in blanketrelevant geometries, such as manifolds, contractions, expansions, bends, etc as also shown in Fig. 1. Although high values of the flow parameters can be achieved in present computations for simple flow geometries (e.g. $\mathrm{Ha} \sim 10^{4}$ in the case of fully developed flows in a duct), computations for complex geometries are still limited to significantly lower values.

Typically, MHD flows in a LM blanket are coupled with heat and mass transfer and demonstrate various unsteady features, including instabilities and MHD turbulence. In addition to the Hartmann number, other important relevant parameters are: the Grashof number that characterizes buoyancy forces relative to inertia forces $G r=g \beta \Delta T L^{3} / v^{2}(\beta$ is the volumetric thermal expansion coefficient, $g$ is acceleration of gravity, and $\Delta T$ is a 
characteristic temperature difference in the fluid), and the hydrodynamic Reynolds number (ratio of inertia to viscous forces) defined through the mean bulk velocity $U_{\mathrm{m}}$ as $R e=U_{\mathrm{m}} L / v$. Present computations for 3D MHD flows with buoyancy forces are limited to $G r \sim 10^{8}$, while the target value for blanket applications is $G r \sim 10^{12}$ [7]. The Hartmann and the Reynolds numbers are often grouped together in the form of the interaction parameter $\mathrm{N}=\mathrm{Ha}^{2} / \mathrm{Re}$, which is the ratio between MHD and inertia forces. Another important parameter is the wall conductance ratio $c_{w}=\sigma_{w} t_{w} / \sigma L$ ( $t_{\mathrm{w}}$ is the wall thickness), which has to be taken into account in MHD flows with electrically conducting walls.

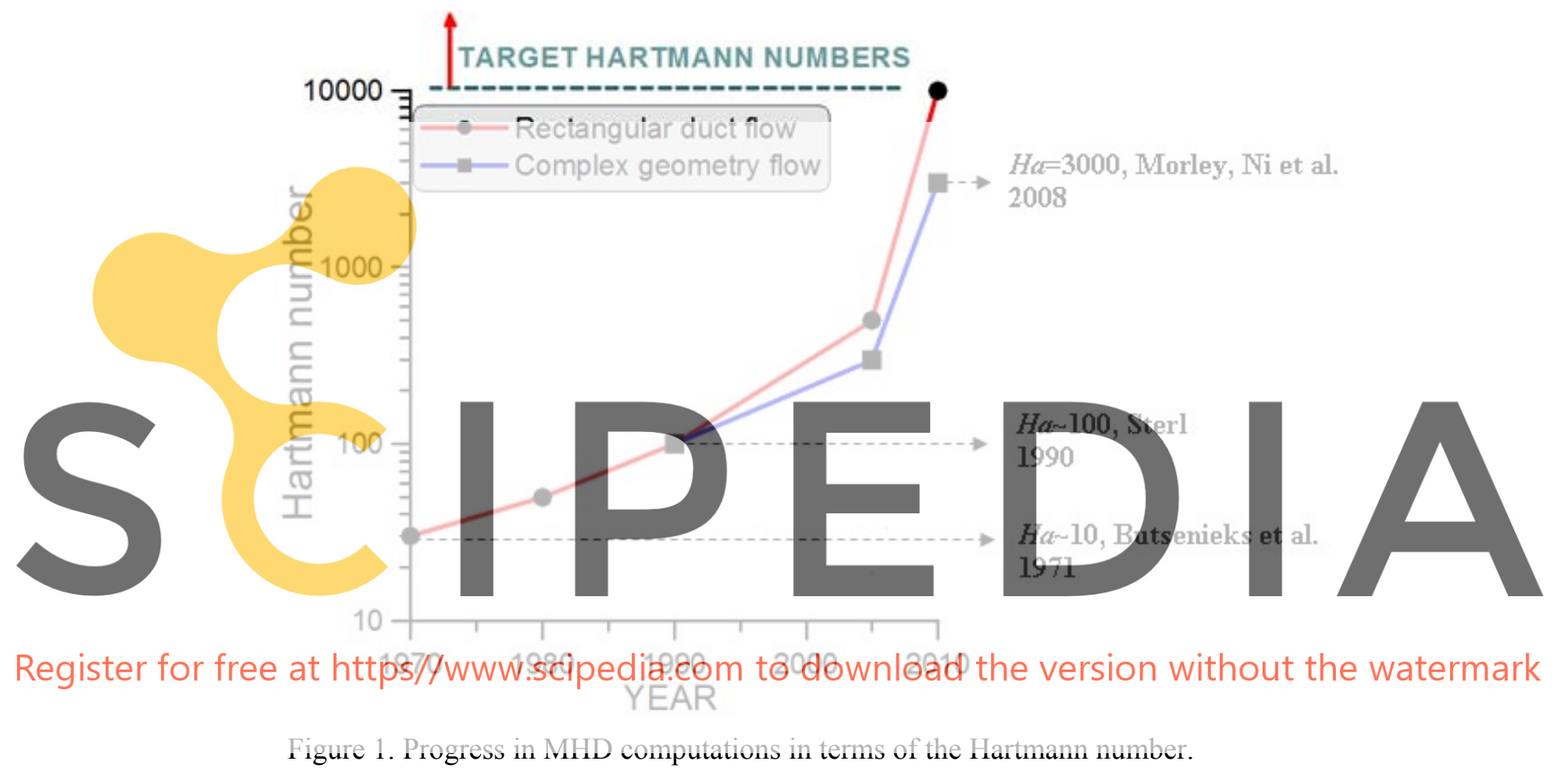

The practical goal on the pathway towards the MHD code, which will fully serve fusion needs on the part of MHD applications, is to achieve high Hartmann $\left(\sim 10^{4}\right)$, Reynolds $\left(\sim 10^{4}\right)$ and Grashof $\left(\sim 10^{12}\right)$ numbers for complex 3D geometries and multimaterial domains [7]. The required codes should allow computations for unsteady (including turbulent) and developing flows and to couple MHD with heat and mass transfer. It is obvious that these goals cannot be realized without intensive code testing, which in turn will require a benchmark database.

\section{Example of code benchmarking from commercial CFD codes}

A useful benchmark exercise for ordinary CFD codes was performed in the recent past as described in [1]. The objective of this exercise was to provide the engineering and scientific community with a common reference point for evaluation of commercial CFD codes. A series of five benchmark simulations was completed using eight commercial CFD codes. These simulations were performed by the vendors themselves, and then reported in CFD forums. The first group of benchmarks consisted of three laminar flow 
problems. These were the steady, 2D flow over a backward-facing step, the low Reynolds number flow around a circular cylinder, and the unsteady 3D flow in a shear-driven cubical cavity. The second group of benchmarks consisted of two turbulent flow problems: first, for unsteady, 2D turbulent flow around a square cylinder, and second, for a 3D spatially-developing flow in a 180-degree bend. All simulation results were evaluated against existing experimental data. Each vendor was given approximately five months to perform the simulations and write a summary paper. Although twenty vendors were invited, only eight vendors agreed to participate in the exercise. Only two vendors completed both laminar and turbulent cases. In general, the codes that completed the first two 2D laminar simulations gave acceptable results, when the codes were applied correctly. However, some of the vendors demonstrated the general lack of insight brought to the solution process. That is, some vendors were satisfied with solutions generated at a single grid resolution, never attempting to demonstrate a grid convergent solution. The third benchmark problem, the unsteady 3D flow in a cavity, requires a spatial discretization scheme that minimizes numerical diffusion. With numerical diffusion present, the moderate scale, secondary motions never develop, i.e., the Görtler vortices. In some of the simulations performed, Görtler vortices were not predicted or sufficiently resolved by the grid. And again, some of the vendors simply did not demonstrate any insight into the solution of the problem. Regarding the two turbulent flow benchmark simulations in this exercise, most of the vendors refused, motivating their decision by the fact that the proposed cases are beyold present CFD-capabilities. Those who performed
the tests demonstrated greater insight into the problem solution and, in some cases did
solve the problems on multiple grids with significantly different resolutions.
4. Review of available MIHD codles utilized by fusion community

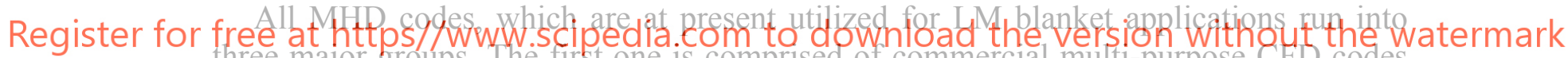
three major groups. The first one is comprised of commercial multi-purpose CFD codes with a built-in or user-defined MHD module. Four typical examples of such codes are FLUENT (now a part of ANSYS), CFX (also a part of ANSYS), SC/TETRA by CRADLE and FLUIDYN by TRANSOFT International. Another code, which can be added to this group, is OpenFOAM, an open-source multi-purpose CFD toolbox with a built-in electromagnetic module developed by OpenCFD Ltd at ESI Group and distributed by the OpenFOAM Foundation. It should be stressed that even though hydrodynamic solvers in the above tools have been brought to the point where they are useful in engineering design, the MHD components of these codes are still not mature and need many improvements.

The second group includes massive non-commercial "home-made" solvers, which are specially developed for MHD applications. Among such codes are HIMAG (USA) [8], MTC (China) [9] and FEMPAR (Spain) [10]. Unlike many commercial codes, these codes are relatively new, have not been fully validated, don't have a convenient user interface and often need to be modified to meet specifications of a particular problem. The advantages of such codes are: their focus on MHD problems and flexibility compared to "black-box" commercial codes when any changes in the source code are required. 
The third group is represented by research codes, which are typically limited to a special type of flow and/or simple flow geometries. Such codes are many in number and can hardly be reviewed in this paper. A few relevant examples are the CoreFlow [11], which can be applied to inertialess flows, research codes based on the asymptotic technique [12] and the TRANSMAG code for analysis of MHD induced corrosion of ferritic steels in the flowing lead-lithium alloy [13]. Many other examples can be found elsewhere. A special group of research codes that address MHD turbulent flows includes Direct Numerical Simulation (DNS) (e.g., [14]) and Large Eddy Simulation (LES) (e.g. [15]).

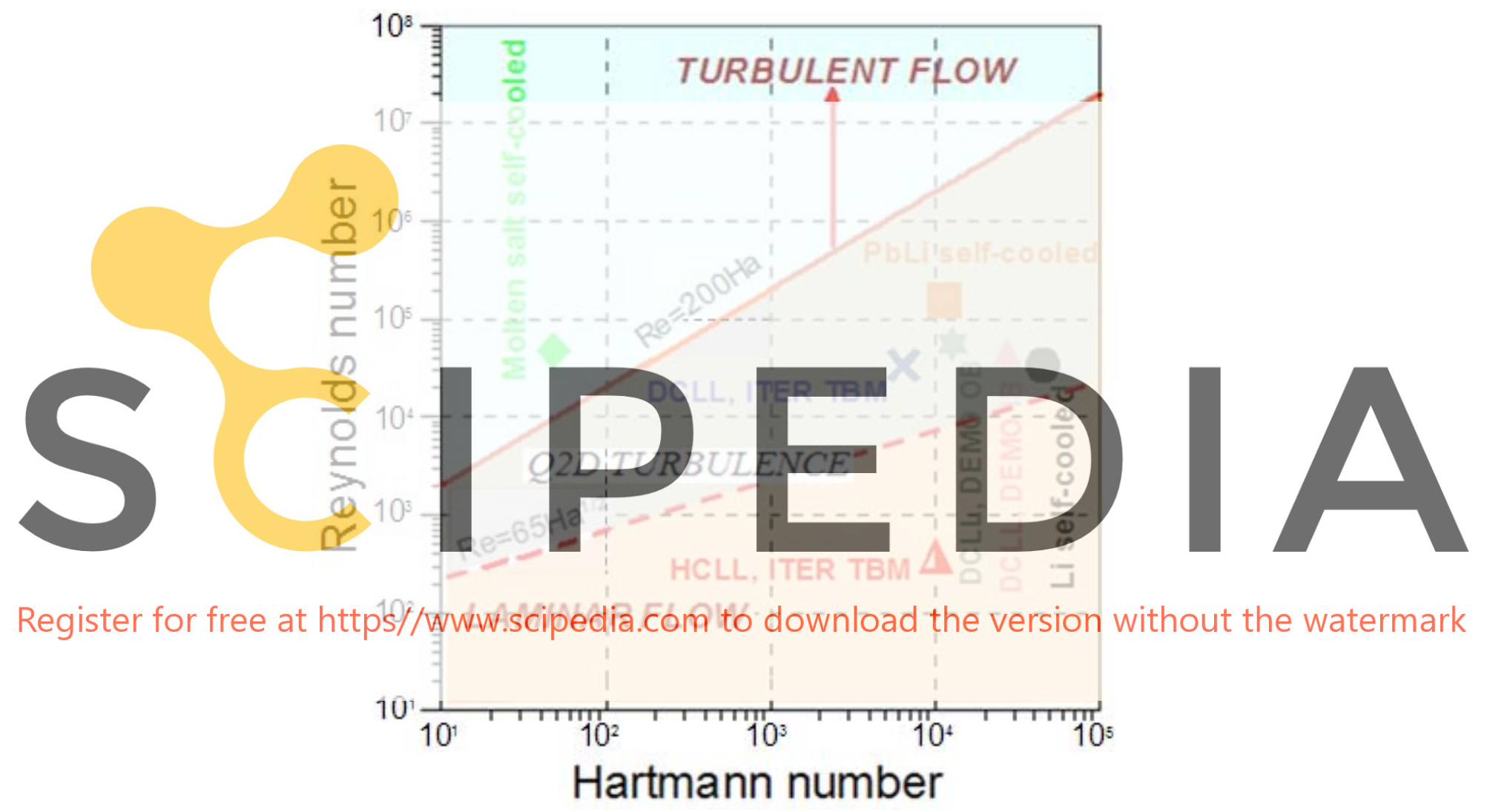

Figure 2. Hartmann - Reynolds number diagram can be used to predict flow regimes in liquidbreeder blankets, including HCLL (ITER TBM), DCLL (ITER TBM and DEMO), Li/V selfcooled, PbLi self-cooled and molten salt self-cooled blankets. In the lower, middle and upper areas on the diagram, blanket flows are seen to be laminar, Q2D turbulent and 3D turbulent, correspondingly.

\section{Selected benchmark problems}

For decades MHD studies of blanket flows were mostly focused on prediction of the MHD pressure drop in typical blanket configurations. Among common concerns of LM blankets, reduction of MHD pressure drop still remains one of the most important issues, stimulating new ideas and efforts on decoupling the electrically conducting wall from the fluid. However, there are many important phenomena beyond the MHD pressure drop 
that have not been fully addressed yet. That is why current studies are focusing more on the detailed structure of MHD flows, including various 3D and unsteady effects associated with buoyancy-driven convection, flow instabilities, and MHD turbulence [16]. These complex MHD processes can affect transport properties of MHD flows in a drastic way and have a profound impact on blanket performance. In fact, in almost all LM blankets, the flows are envisaged to demonstrate instabilities and eventually turbulence. In high Hartmann, high Reynolds number MHD flows, the latter takes a special form of quasi-two-dimensional (Q2D) turbulence [17] as illustrated in Fig. 2 for a relatively simple fully developed flow in a non-conducting square duct in a transverse magnetic field. Therefore, the selected benchmarks should address a wide spectrum of MHD flows from laminar to turbulent and Q2D turbulent flows. To span this variety of flows, we recommend here five benchmark problems (A-E): two for laminar flows, two for turbulent/Q2D flows and one for MHD flows with heat transfer. These five benchmark problems are described below.

\section{Benchmark problem A. Fully developed laminar steady MHD flow}

\section{The recommended benchmark for fully developed flows is a classic 2D MHD problem} for a flow of electrically conducting fluid in a rectangular duct subject to a uniform transverse magnetic field. This problem comes back to studies by Shercliff [18] for a

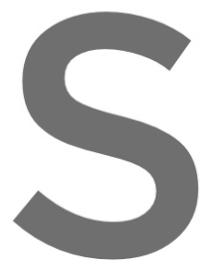
non-conducting duct both cases the flow field with the thick the magnetic field uniform. If the duct walls near the side walls also scaling in width as $1 / \mathrm{Ha}^{0.5}$ ("M-shaped" velocity profile). Two

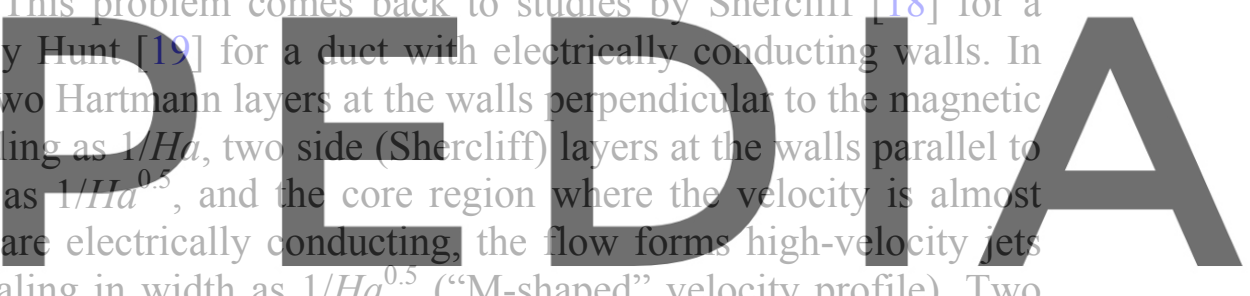

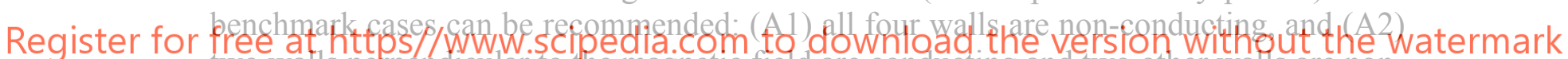
two walls perpendicular to the magnetic field are conducting and two other walls are nonconducting.

Table I. Dimensionless flow rate $\tilde{Q}$ computed with analytical solutions in [18] and [19] and numerically using an MHD code in Ref. [20].

\begin{tabular}{|l|c|c|c|c|c|}
\hline \multicolumn{1}{|c|}{ FLOW } & $H a$ & $\begin{array}{c}c_{\mathrm{w}} \\
\text { Hartmann wall }\end{array}$ & $\begin{array}{c}c_{\mathrm{w}} \\
\text { Side wall }\end{array}$ & $\begin{array}{c}\tilde{Q} \\
\text { Analytical }\end{array}$ & $\begin{array}{c}\tilde{Q} \\
\text { Numerical }\end{array}$ \\
\hline $\begin{array}{l}\text { Non-conducting } \\
\text { duct (A1) }\end{array}$ & 500 & 0 & 0 & $7.680 \times 10^{-3}$ & $7.679 \times 10^{-3}$ \\
\cline { 2 - 6 } & 5000 & 0 & 0 & $7.902 \times 10^{-4}$ & $7.902 \times 10^{-4}$ \\
\cline { 2 - 6 } & 10,000 & 0 & 0 & $3.965 \times 10^{-4}$ & $3.965 \times 10^{-4}$ \\
\cline { 2 - 6 } & 15,000 & 0 & 0 & $2.648 \times 10^{-4}$ & $2.648 \times 10^{-4}$ \\
\hline $\begin{array}{l}\text { Conducting duct } \\
\text { (A2) }\end{array}$ & 500 & 0.01 & 0 & $1.405 \times 10^{-3}$ & $1.401 \times 10^{-3}$ \\
\cline { 2 - 6 } & 5000 & 0.01 & 0 & $1.907 \times 10^{-5}$ & $1.896 \times 10^{-5}$ \\
\cline { 2 - 6 } & 10,000 & 0.01 & 0 & $5.169 \times 10^{-6}$ & $5.139 \times 10^{-6}$ \\
\hline
\end{tabular}




\begin{tabular}{|l|l|l|l|l|l|}
\hline & 15,000 & 0.01 & 0 & $2.425 \times 10^{-6}$ & $2.404 \times 10^{-6}$ \\
\hline
\end{tabular}

The numerical data for the dimensionless flow rate $\tilde{Q}=\int_{-1}^{1} d \tilde{y} \int_{-1}^{1} \tilde{U} d \tilde{z}$ computed from the analytical solutions in [18] and [19] for a square duct for several Hartmann numbers from 500 to 15,000 are summarized in Table I. The dimensionless axial velocity in the formula for the flow rate is $\tilde{U}=U /\left[b^{2} v^{-1} \rho^{-1}(-d P / d x)\right]$, where $-d P / d x$ is the pressure gradient in the liquid. The cross sectional coordinates $z$ (along the magnetic field) and $y$ (across the magnetic field) are scaled by $b$ and by $a$ (half of the duct size in the direction perpendicular to the magnetic field): $\tilde{z}=z / b$ and $\tilde{y}=y / a$. Being an integral quantity, $\tilde{Q}$ is better suited as a comparison parameter than the local velocities. Some computations were already done using a research code [20] on a stretched mesh of the size 99x99, which provides clustering mesh points near the walls for proper resolution of the Hartmann and side layers. In these computations, the convergence criterion was $\left|\left(\tilde{Q}^{n+1}-\tilde{Q}^{n}\right) / \tilde{Q}^{n}\right|<10^{-10}$, where $n$ is the iteration number. As seen from Table I, the computations are in very good agreement with the analytical solution.

\section{Benchmark problem B. 3D laminar steady MHD flow}
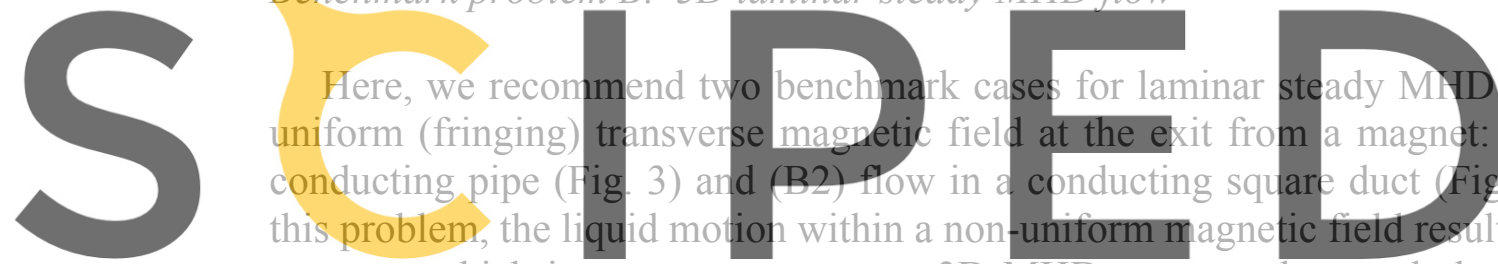

\section{this problem, the liquid motion within a non-uniform magne}

currents, which in turn cause an extra 3D MHD pressure drop and change the velocity

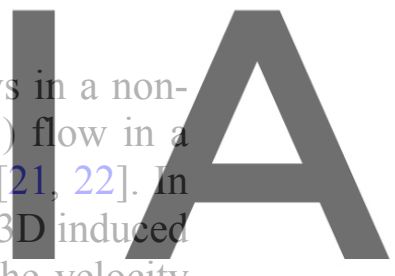

Register for free at https//www.scipedia.com to download the version without the watermark

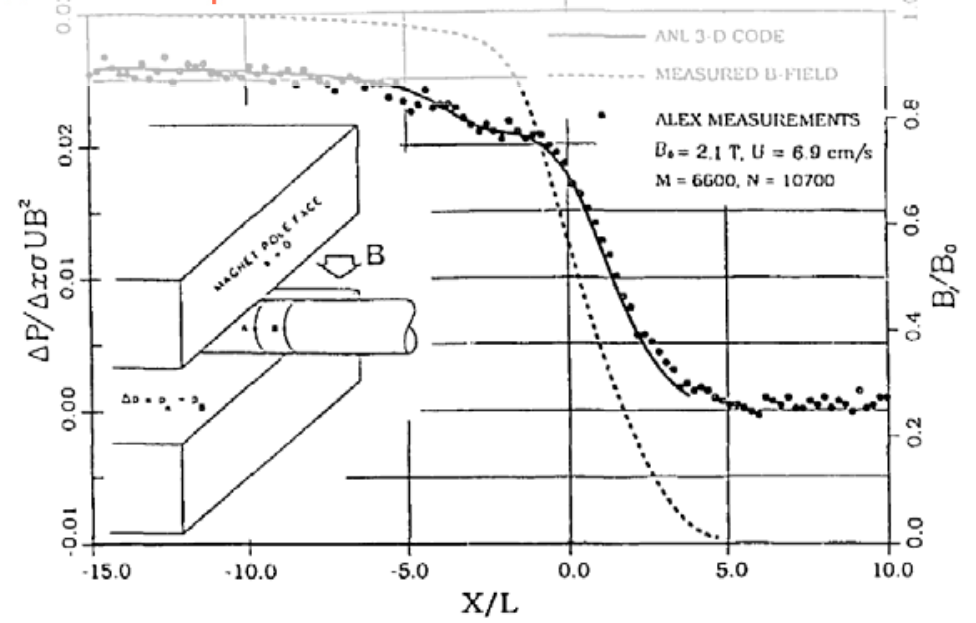

Figure 3. Benchmark case B1 for a pipe flow in a fringing magnetic field [21]. The figure shows the magnetic field distribution and the pressure drop measured between two points $\mathrm{A}$ and $\mathrm{B}$. The dimension $L$ is the pipe radius.

The pressure drop data have been accurately documented and can be used for code benchmarking. The dimensionless flow parameters matching those in the experimental 
studies in the aforementioned references for eutectic sodium potassium alloy (NaK) flows in a magnetic field up to $2.1 \mathrm{~T}$ are summarized in Table II. Dimensional data can be found in $[21,22]$.

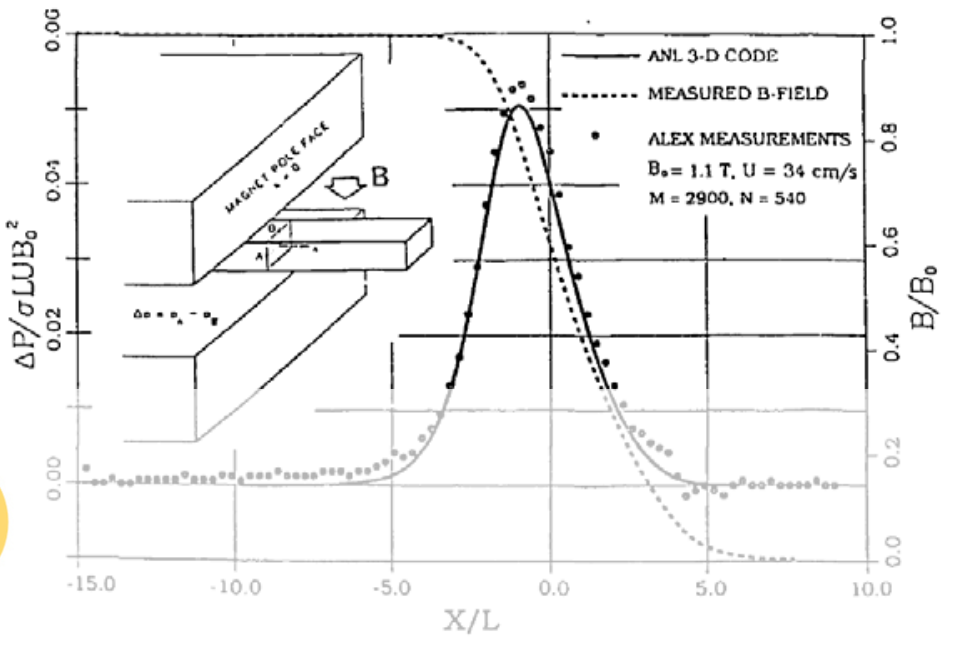

Figure 4. Benchmark case B2 for a square duct flow in a fringing magnetic field [21].

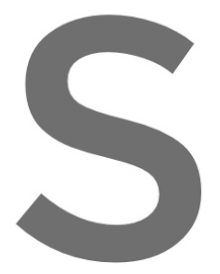

The figure show between two point

Table II. Dimension Flow Pipe (B1)
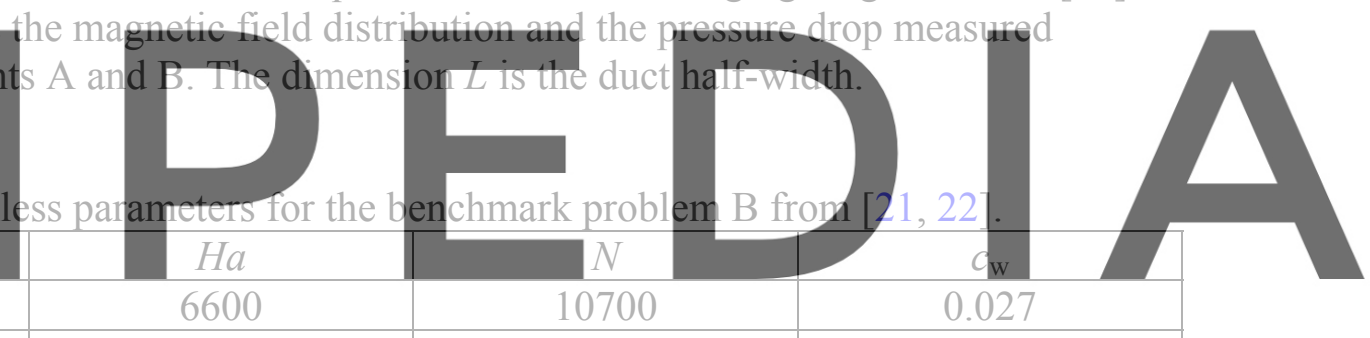

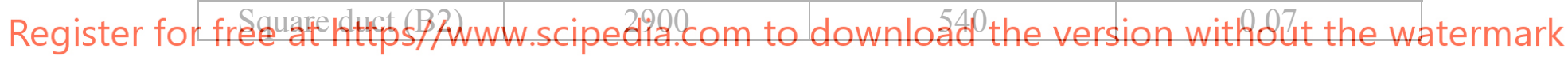

\section{Benchmark problem C. Q2D turbulent MHD flow}

The suggested benchmark problem for this class of MHD flows has been adopted from the experimental study [23] where Q2D turbulent $\mathrm{NaK}$ flows in a thin-wall conducting rectangular duct (Fig. 5) were observed for Hartmann numbers from 600 to 4800 and for Reynolds numbers from $3.3 \times 10^{3}$ to $1.0 \times 10^{5}$. The instabilities and associated turbulence transitions in these flows are related to formation of the M-shaped base velocity profile with two inflection points.

Table III. Dimensionless parameters for the benchmark problem C [23].

\begin{tabular}{|c|c|c|c|c|c|}
\hline Case & $b / a$ & $H a$ & $R e$ & $c_{\mathrm{W}}($ side wall) & $c_{\mathrm{W}}$ (Hartmann wall) \\
\hline C1 (Type I) & 2 & 4800 & $3 \times 10^{4}$ & 0.0714 & 0.0119 \\
\hline C2 (Type II) & 2 & 1200 & $1 \times 10^{5}$ & 0.0714 & 0.0119 \\
\hline
\end{tabular}

Two Q2D turbulence regimes have been identified in this study. Using the original terminology [23], Type I instability occurs in the outer, bulk-side shear layer of the sidewall jet, while in Type II instability turbulent vortices are generated in the inner shear layer. More recent studies of instabilities and turbulence transitions in Q2D flows with a 
side-wall jet [24] suggest new interpretation of the observed instability types. Namely, Type I instability can be interpreted as a primary Kelvin-Helmholtz instability in the bulk flow associated with the inflection point in the velocity profile, while Type II instability seems to be related to destabilization of the side boundary layer by the bulk vortices. In accordance with the observed two turbulent flow regimes, two benchmark cases $\mathrm{C} 1$ (Type I instability) and C2 (Type II instability) are summarized in Table III.

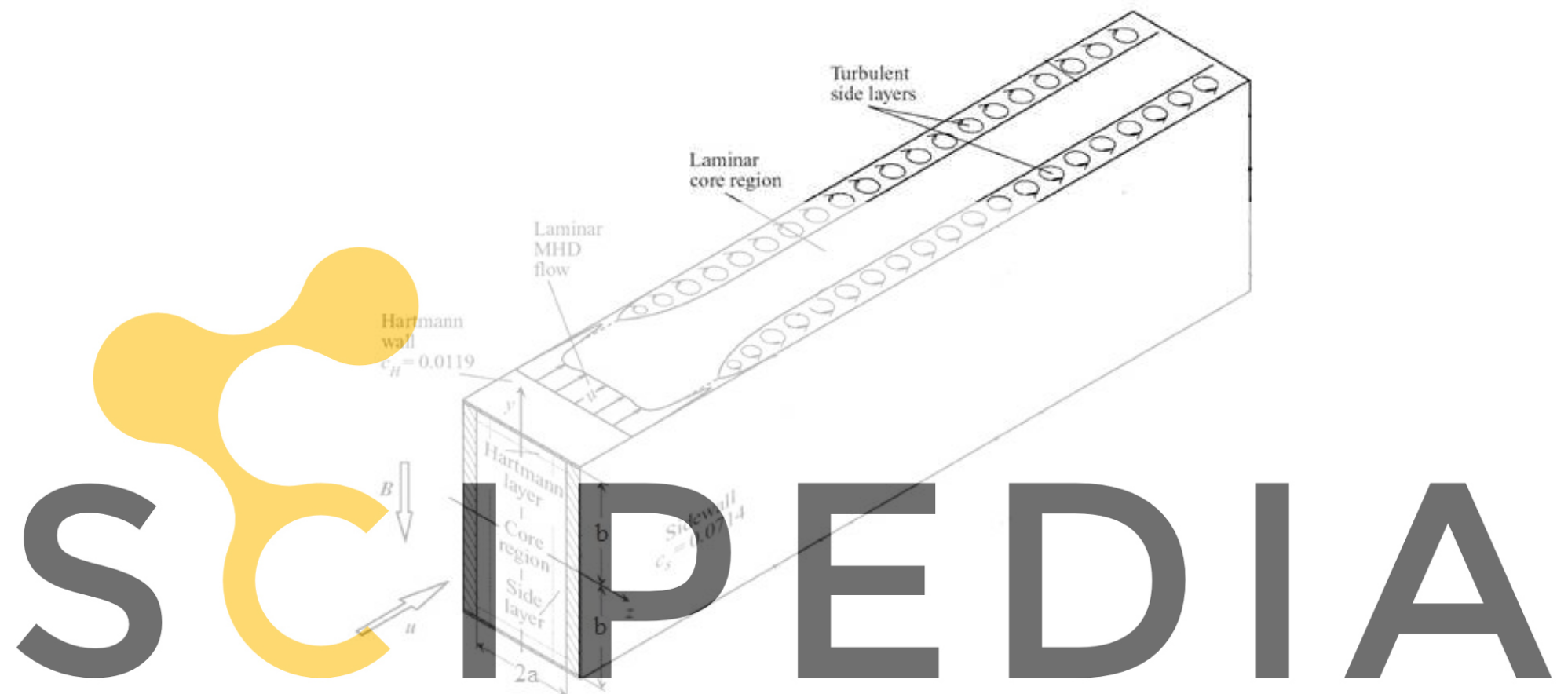

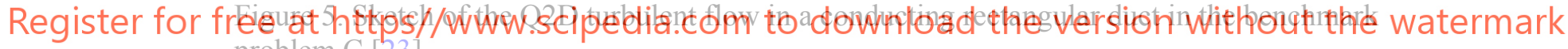
problem C [23].
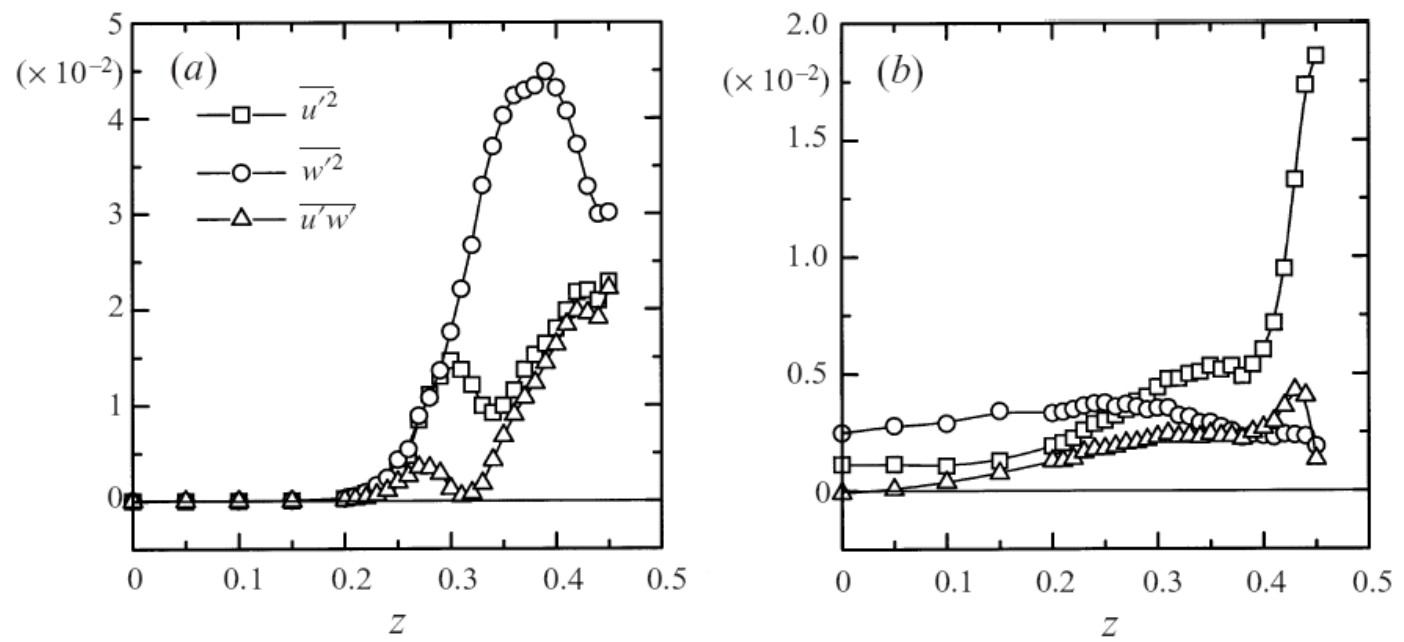

Figure 6. Q2D turbulent flow in a conducting duct (Benchmark problem C). Distribution of Reynolds stresses in the midplane $(y=0)$ along the $z$-direction: (a) Type I instability $(H a=4800$, $\left.R e=3 \times 10^{4}\right)$, and (b) Type II instability $\left(H a=1200, R e=1 \times 10^{5}\right)$. The velocity components are scaled with the mean bulk velocity. 
To our best knowledge, there have been no successful efforts to reproduce experimental results in [23] in computations up to now. Taking into account the significant computational challenge, any computations that can reproduce the abovementioned flow regimes should be considered as successful. A significant amount of statistical data, which can be used for benchmarking the codes can be found in [23], including Reynolds stresses, turbulent kinetic energy, and the time averaged velocity profile and pressure drop coefficient. The Reynolds stresses for the benchmark cases $\mathrm{C} 1$ and $\mathrm{C} 2$ are shown in Fig. 6.

\section{Benchmark problem D. MHD turbulent flow}

Two computational techniques presently used for 3D MHD turbulent flows are DNS (Direct Numerical Simulation) and LES (Large Eddy Simulation). Both approaches are suggested for participation in the benchmarking study. It is also suggested that the analysis is limited to the most thoroughly studied configuration, namely the flow in a duct of rectangular cross-section with electrically insulating walls. This flow, while simple, combines the essential features of the transformation in response to the magnetic field: modification of mean velocity, suppression of fluctuations, and development of Hartmann and Shercliff boundary layers. Two archetypal cases can be considered: (D1) that of a uniform transverse (parallel to a set of walls) magnetic field imposed on a long section of the duct and (D2) that of a 'magnetic obstacle', which is an area of localized transverse magnetic field created by short pole-pieces of a permanent magnet.

In both cases, it would be interesting, in addition to the variables computed directly for the benchmarking, to consider visualizations of instantaneous and time-averaged velocity fields.

\section{(D1) Uniform magnetic field}

In this case, the accuracy of simulations is largely determined by the ability of the numerical model to correctly describe the anisotropic suppression of turbulent fluctuations and transformation of the mean flow (see, e.g. [14,15,25]). One should also consider transitional laminar-turbulent flows, which are of special interest. It should be assured that the model correctly reproduces the peculiar flow features at $\mathrm{R}=\mathrm{Re} / \mathrm{Ha}$ between approximately 200 and 500, such as localization of turbulence in sidewall layers $[14,15]$ and formation of isolated turbulent spots [26].

Table IV. Parameters of the benchmark problem D1 for turbulent flow in a duct with uniform magnetic field [27]. The Reynolds and Hartmann numbers are based on the hydraulic diameter and mean velocity as the typical scales.

\begin{tabular}{|l|l|l|l|l|}
\hline$R e$ & $(H a / R e) \times 10^{4}$ & DNS/LES & $\begin{array}{l}\text { Variables to be } \\
\text { compared }\end{array}$ & $\begin{array}{l}\text { Figures in } \\
{[27]}\end{array}$ \\
\hline 53000 & $\begin{array}{l}5,9.5,10.7,13.9, \\
18,19,21,24,25.3\end{array}$ & both & $\begin{array}{l}\text { Friction coefficient } C \mathrm{f} \\
\text { Mean velocity } U \\
\text { rms fluctuations } u^{\prime}, v^{\prime} \\
\text { Reynolds stress }\end{array}$ & $\begin{array}{l}1,3,7,15,22 \\
16,22\end{array}$ \\
\hline
\end{tabular}


The experiment [27] is suggested as a benchmark. In this experiment, flow in a duct of aspect ratio 5.82 is subject to a uniform magnetic field on the length of 21 hydraulic diameters. The magnetic field is parallel to the shorter walls. A computational model can assume a fully developed state and use the standard formulation with relatively short computational domain, periodic inlet-exit conditions, and the uniform one-component magnetic field applied in the entire domain. The suggested parameters of the simulations are listed in Table IV.

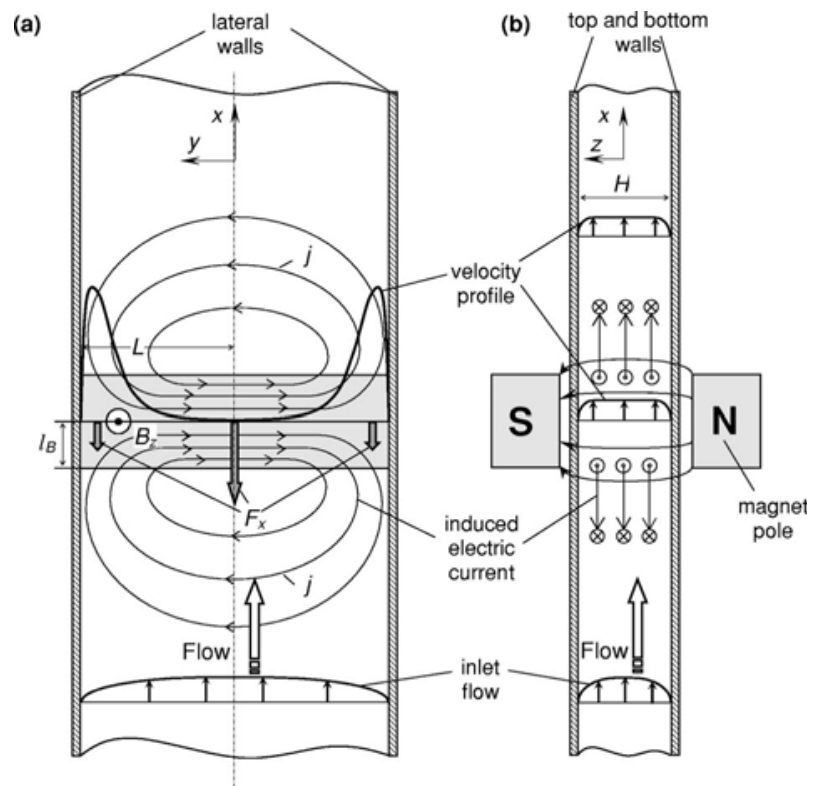

Figure 7. Flow configuration for the benchmark problem D2 (turbulent flow in a duct with magnetic obstacle [28]).

\section{D2. Magnetic obstacle}

Here, the main features of the flow are the suppression of turbulence upon entering the magnetic field, formation of large-scale vortices under the influence of the magnet, and development of long turbulent jets near the walls parallel to the magnetic field. A good computational model should be able to accurately reproduce all of them. It is possible that a combination of turbulent and nearly laminar zones makes this problem particularly challenging for LES. For the benchmarking, the results of the experiment [28] conducted at $R e=4000, H a=400$ and duct aspect ratio 5 can be used. The magnetic field is localized with the main component parallel to the shorter walls. The non-dimensional parameters are based on the maximum magnetic field, shorter wall size, and mean flow velocity as the typical scales. Ref. [28] provides measurements of profiles of mean velocity $U$ and intensity of streamwise velocity fluctuations at two locations downstream of the magnet (see Fig. 8 of [28]) that can be used for verification and validation purposes. 


\section{Benchmark problem E. MHD flow with heat transfer}

The suggested benchmark problem is based on experimental studies [29] of MHD buoyancy-driven convection for a mercury flow in a differentially heated vertical enclosure of a square cross-section [40 x 40 x $300(\mathrm{~mm})]$. The applied transverse magnetic field in the experiment is perpendicular to the temperature gradient (Fig. 8). The natural convection occurs due to the imposed temperature difference between two vertical walls. These two walls (hot and cold) are 1-cm thick copper plates kept at constant temperatures $T_{\mathrm{h}}$ and $T_{\mathrm{c}}$ by strong water flows. All other walls are made of Plexiglas, i.e., electrically and thermally insulating. The copper plates are coated with epoxy in order to insulate them electrically from mercury.

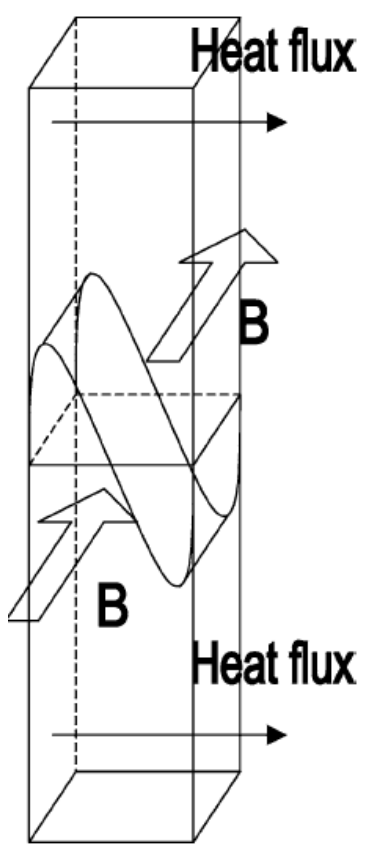

(a)

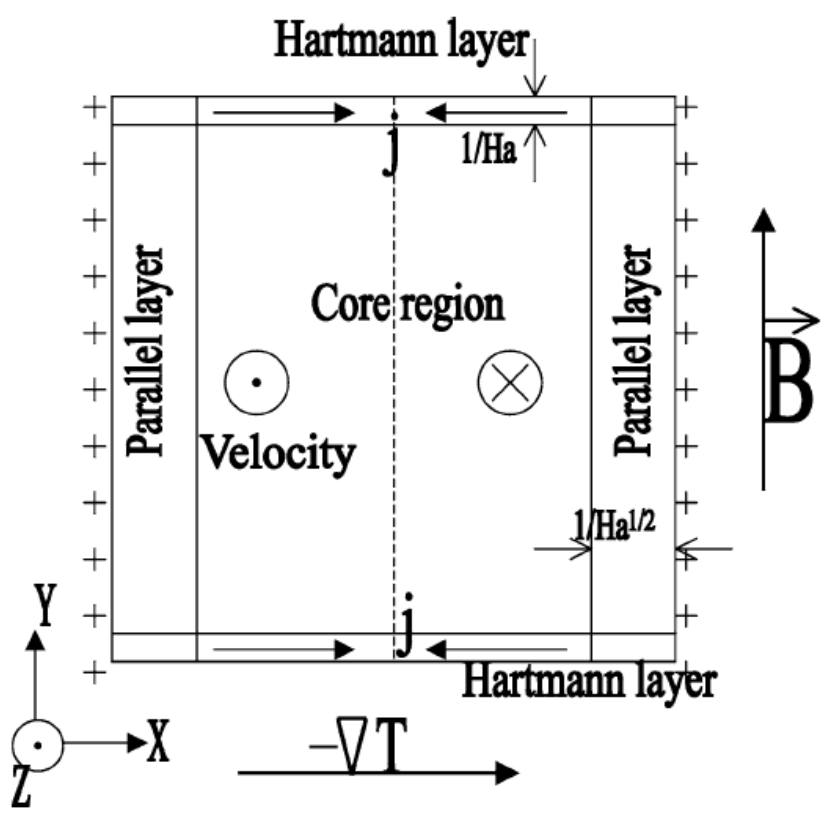

(b)

Figure 8. Benchmark Problem D on MHD flows with heat transfer. Schematics of the experimental setup in [29] for MHD natural-convection flows: (a) vertical enclosure, (b) crosssectional area.

Relevant dimensionless parameters are the Hartmann number and the Grashof number, which are constructed in [29] using the entire cross-sectional duct dimension $2 b$. For this benchmark problem, the main goal is to reproduce in computations the experimental Nusselt number $(\mathrm{Nu})$ versus $\mathrm{Ha}$ as shown in Fig. 9. Suggested parameters for computations are 100, 200, 300, 400, 500, 600, 700, 800 for the Hartmann number and $3 \times 10^{7}$ and $1.5 \times 10^{8}$ for the Grashof number. The Nusselt number is defined as

$N u=\frac{\int_{(S)} q^{\prime \prime} d s}{k_{H g}\left(T_{h}-T_{c}\right) 2 b}$ 
In this formula, the numerator is the total heat applied to the hot wall (or extracted from the cold wall) and the denominator represents conduction heat transfer between the hot and cold walls. As seen from Fig.9, the Nusselt number demonstrates a peak at Ha 200300 .

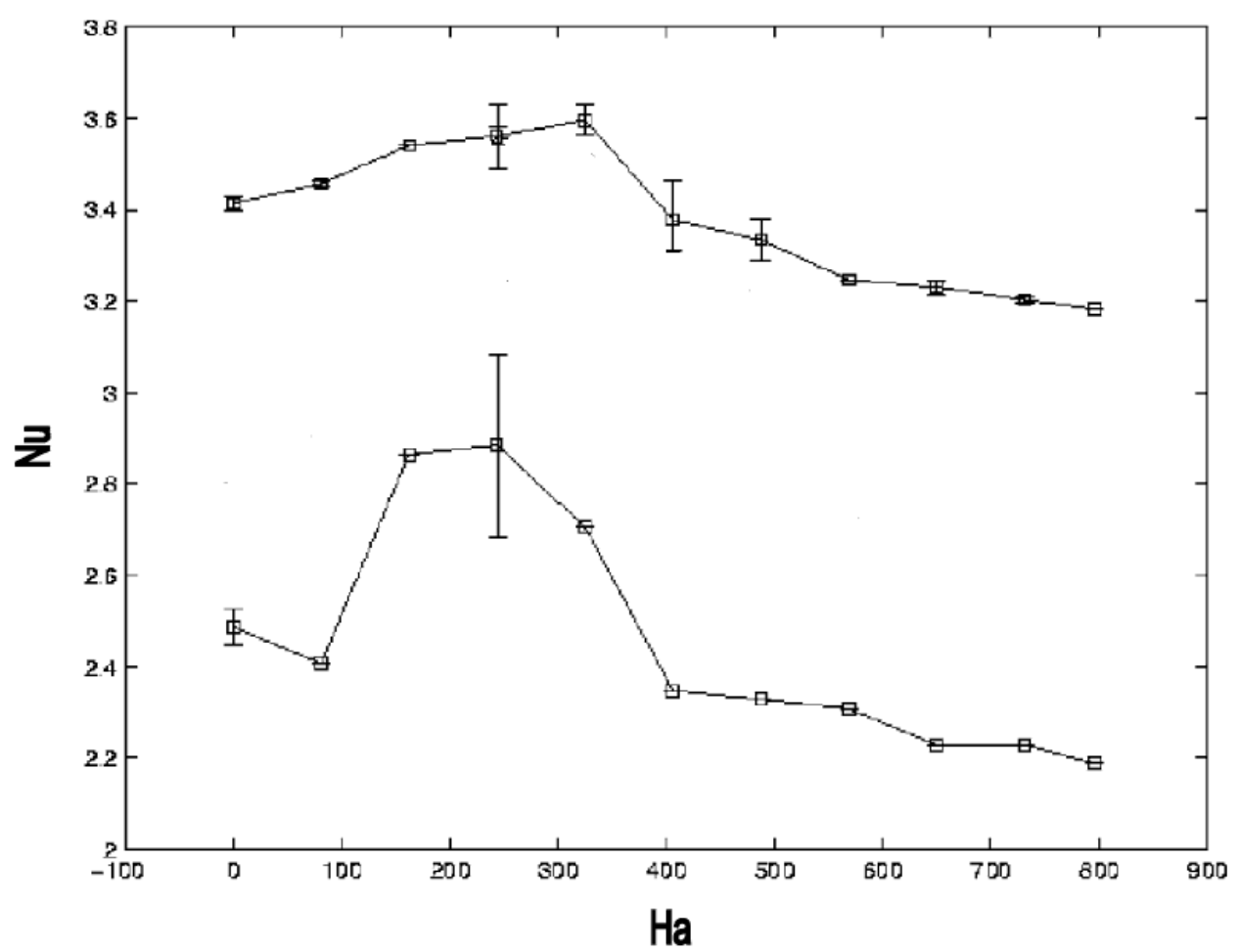

Figure 9. Nusselt number as a function of Hartmann number in the naturalconvection flow studied in [29].

The 3D computations, also presented in [29], predict the location of the peak at $H a \sim 200$ but the computed Nusselt numbers are higher. The differences can be related to both the experiment and the computations. As alternative benchmarks, one can consider experiments on MHD natural-convection flows in [30] and [31].

\section{Other code testing}

The benchmark problems recommended above are limited to Hartmann numbers not higher than a few thousands, i.e., considerably lower than those in fusion applications. This limitation is due to the fact that typical laboratory magnets have maximum magnetic fields of less than $2 \mathrm{~T}$. Of all selected benchmark problems, only problem A, for which comparisons are made against an analytical solution, proposes high Hartmann numbers up to 15,000 . Therefore, upon successful accomplishment of the proposed benchmark cases, the code developers/users can further be motivated to perform more code testing at higher flow parameters. Unfortunately, for very high $\mathrm{Ha}$, Re and $\mathrm{Gr}$ experimental data are not available, so that code-to-code comparisons remain the only possible approach to 
address the validity of computations. Such efforts on extending the code capabilities towards the realistic fusion range are highly encouraged but are not considered as a part of the present approach to verification and validation of MHD codes for fusion applications.

In fact, all above recommended benchmarks are based on simple flow geometries, i.e., a straight rectangular duct or a pipe of constant cross-section. In any typical blanket module, the geometry is more complex, involving contractions, subtractions, bends, manifolds, etc. The duct flows in a blanket are often coupled electromagnetically giving rise to higher MHD pressure drop (Madarame effect). It is useful to have benchmark problems of this type too. As possible candidates for benchmarks of this type one can consider experiments on laminar MHD flows in a Mock-Up of HCLL blanket [32] or experimental studies of MHD flows in a sudden expansion [33]. However, taking into account a significant number of already proposed benchmarks, we recommend these two problems to be considered as a next step providing that problems A- D are accomplished.

\section{Recommendations on how to organize $V \& V$}

Our recommendations are based mostly on experience, which can be gained from the already mentioned benchmark activities for CFD codes described in [1]. Taking into account complexity, uniqueness and computational challenges of proposed benchmark problems for CMHD, all participants will be given sufficient time, a year, to accomplish their computations. The benchmarks can be performed in any order or even in parallel but it appears to be more effective to start with the simplest, best understood problem for fully developed flows and then to proceed with others as ordered in Section 5, assuming that each problem will take on average two months. Once a particular benchmark is completed, the obtained results can be discussed among the participants before moving to the next one. After completing all the cases, the participants will be given another two months to prepare a report summarizing their findings that should include at least comparisons with an analytical solution (problem A), with experimental data (problems B, $\mathrm{C}, \mathrm{D}$ and $\mathrm{E}$ ), with numerical data (problem E), and also code-to-code comparisons (all problems). The graphical data in Figs. 3, 4, 6 and 9, which should be used for code benchmarking, have been digitized and the data files are available upon request from the first author. Finally, the reports will be compiled in paper/papers to summarize the most important results and conclusions.

When doing computations, the performers are not supposed to use the same computational settings and should be flexible in their decisions about computational parameters, meshes, boundary conditions and closing relations (if there is more than one choice). For example, computations for the case of a developing MHD flow in a fringing magnetic field (Problem B) can be done using only one, transverse, component of the applied magnetic field or an entire magnetic field reconstructed from the transverse component such that the magnetic field satisfies the divergence- and curl-free requirements. A reconstruction procedure has been discussed for instance in [34]. Another example is LES of turbulent flows (Problem D), where several decisions could be made about the subgrid-scale model, e.g., Smagorinsky versus dynamic model. Also, in the case of electrically conducting ducts, it needs to be decided if the thin-wall 
boundary condition is sufficient or the alternative approach of solving the electromagnetic equations within the conducting wall is required. While making such decisions, the participants are responsible for all necessary tests to make sure that their computations adequately address a problem. This also includes mesh sensitivity and convergence analysis and other code testing that may require other benchmarks in addition to those proposed in this paper. All such details about computational settings and extra testing are certainly of a significant interest to all participants and have to be included in the reports. The CPU time, memory usage and the ability of a code to use effectively the computational resources, i.e. scalability and parallelization, is important information that should also be reported for each simulation. The principal goal of the proposed testing is, however, not to minimize the CPU time or/and memory usage but to demonstrate the code ability to reproduce accurately experimental or trusted numerical data.

\section{References}

1. C. J. Freitas, Perspective: selected benchmarks from commercial CFD codes, J. Fluids Eng. 117 (1995) 208-218.

2. AIAA, Guide for the Verification and Validation of Computational Fluid Dynamics Simulations, AIAA G-077-1998e, 1998.

3. I. E. Butsenieks, G. A. Vitolin'sh, Yu. M. Gel'fgat, V. S. Dorofeev, E. V. Shcherbinin, Magnetohydrodynamic flow in a square tube with walls of different electrical conductivity in an oblique transverse magnetic field, Magnetohydrodynamics 3 (1971) 53-60.

4. A. Sterl, Numerical simulation of liquid-metal MHD flows in rectangular ducts, J. Fluid Mech. 216, 161-191 (1990).

5. N. B. Morley, M.-J. Ni, R. Munipalli, P. Huang, M. A. Abdou, MHD simulations of liquid metal flow through a toroidally oriented manifold, Fusion Eng. Des. 83 (2008) 1335-1339.

6. M.-J. Ni, R. Munipalli, N.B. Morley, P. Huang, M.A. Abdou, A current density conservative scheme for incompressible MHD flows at a low magnetic Reynolds number. Part I: on a rectangular collocated grid system, J. Comp. Phys. 227 (2007) 174-204.

7. S. Smolentsev, R. Moreau, L. Bühler, C. Mistrangelo, MHD thermofluid issues of liquid-metal blankets: phenomena and advances, Fusion Eng. Des. 85 (2010) 1196-1205.

8. S. Smolentsev, N. B. Morley, M. Abdou, R. Munipalli, R. Moreau, Current approaches to modeling MHD flows in the dual coolant lead lithium blanket, Magnetohydrodynamics 42 (2006) 225-236.

9. T. Zhou, Z. Yang, M.-J. Ni, H. Chen, Code development and validation for analyzing liquid metal MHD flow in rectangular ducts, Fusion Eng. Des. 85 (2010) 1736-1741.

10. R. Planas, S. Badia, R. Codina, Approximation of the inductionless MHD problem using a stabilized finite element method, J. Comp. Phys. 230 (2011) 2977-2996. 
11. L. Bühler, Magnetohydrodynamic flows in arbitrary geometries in strong, nonuniform magnetic fields - a numerical code for the design of fusion reactor blankets, Fusion Tech. 27 (1995) 3-24.

12. S. Molokov, Liquid metal flows in insulating elements of self-cooled blankets, Fusion Eng. Des. 27 (1995) 642-649.

13. S. Smolentsev, S. Saeidi, S. Malang, M. Abdou, Numerical study of corrosion of ferritic/martensitic steels in the flowing $\mathrm{PbLi}$ with and without a magnetic field, J. Nuclear Materials 432 (2013) 294-304.

14. D. Krasnov, O. Zikanov, T. Boeck, Numerical study of magnetohydrodynamic duct flow at high Reynolds and Hartmann numbers, J. Fluid Mech. 704 (2012) 421-446.

15. H. Kobayashi, Large eddy simulation of magnetohydrodynamic turbulent duct flows, Phys. Fluids 20 (2008) 015102.

16. S. Smolentsev, N. Vetcha, M. Abdou, Effect of a magnetic field on stability and transitions in liquid breeder flows in a blanket, Fusion Eng. Des. 88 (2013) 607610.

17. J. Sommeria, R. Moreau, Why, how and when MHD turbulence becomes twodimensional, J. Fluid Mech. 118 (1982) 507-518.

18. J. A. Shercliff, Steady motion of conducting fluids in pipes under transverse magnetic fields, Proc. Camb. Phil. Soc. 49 (1953) 136-144.

19. J. C. R. Hunt, Magnetohydrodynamic flow in rectangular ducts, J. Fluid Mech., 21 (1965) 577-590.

20. S. Smolentsev, N. Morley, M. Abdou, Code development for analysis of MHD pressure drop reduction in a liquid metal blanket using insulation technique based on a fully developed flow model, Fusion Eng. Des. 73 (2005) 83-93.

21. B.F. Picologlou, C.B. Reed, Experimental investigation of 3-D MHD flows at high Hartmann number and interaction parameter, IUTAM Symposium on liquid metal MHD, Riga, USSR, May 16, 1988.

22. C.B. Reed, B.F. Picologlou, T.Q. Hua, J.S. Walker, Alex results - a comparison of measurements from a round and a rectangular duct with 3-D code predictions, IEEE 12 Symposium on Fusion Engineering, Monterey, California, October 12$16,1987$.

23. U. Burr, L. Barleon, U. Müller, A. Tsinober, Turbulent transport of momentum and heat in magnetohydrodynamic rectangular duct flow with strong sidewall jets, J. Fluid Mech. 406 (2000) 247-279.

24. S. Smolentsev, N. Vetcha, R. Moreau, Study of instabilities and transitions for a family of quasi-two-dimensional magnetohydrodynamic flows based on a parametric model, Phys. Fluids 24 (2012) 024101.

25. R. Chaudhary, R., S. P. Vanka, S. P. \& B. G. Thomas, Direct numerical simulations of magnetic field effects on turbulent flow in a square duct. Phys. Fluids 22, (2010) 075102.

26. D. Krasnov, A. Thess, T. Boeck, Y. Zhao, O. Zikanov, Patterned turbulence in liquid metal flow: computational reconstruction of the Hartmann experiment. Phys. Rev. Lett. 110 (2013) 084501.

27. C. Reed, P. S. Lykoudis, The effect of transverse magnetic field on shear turbulence. J. Fluid Mech. 89 (1978) 147-171. 
28. O. Andreev, Yu. Kolesnikov, A. Thess, Experimental study of liquid metal channel flow under the influence of a nonuniform magnetic field. Phys. Fluids 18 (2006) 065108.

29. G. Authié, T. Tagawa, R. Moreau, Buoyant flow in long vertical enclosures in the presence of a strong horizontal magnetic field. Part 2. Fimite enclosures, European J. Mech. B/Fluids 22 (2003) 203-220.

30. U. Burr, L. Barleon, P. Jochmann, A. Tsinober, Magnetohydrodynamic convection in a vertical slot with horizontal magnetic field, J. Fluid Mech. 475 (2003) 21-40.

31. O. Zikanov, Ya.I. Listratov, V.G. Sviridov, Natural convection in horizontal pipe flow with a strong transverse magnetic field, J. Fluid Mech. 486 (2013) 486-516.

32. K. Starke, L. Bühler, S. Horanyi, Experimental MHD - flow analyses in a mockup of a test blanket module for ITER, Fusion Eng. Des. 84 (2009) 1794-1798.

33. L. Bühler, S. Horanyi, E. Arbogast, Experimental investigation of liquid-metal flows through a sudden expansion at fusion-relevant Hartmann numbers, Fusion Eng. Des. 82 (2007) 2239-2245.

34. X. Albets-Chico, E.V. Votyakov, H. Radhakrishnan, S. Kassinos, Effects of the consistency of the fringing magnetic field on direct numerical simulations of liquid-metal flow, Fusion Eng. Des. 86 (2011) 5-14. 